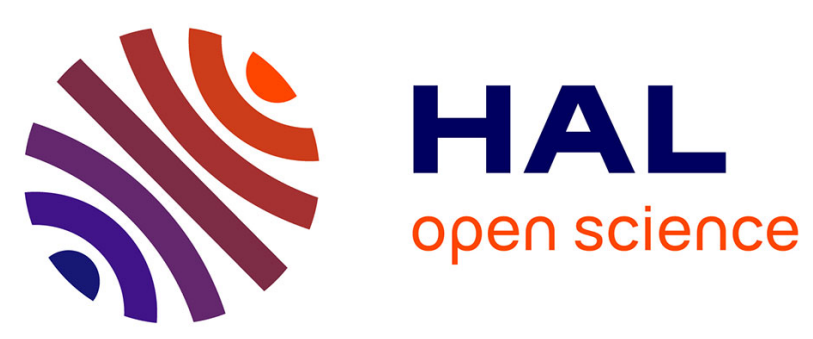

\title{
Energy of a single bead bouncing on a vibrating plate: Experiments and numerical simulations
}

Jean-Christophe Géminard, C Laroche

\section{To cite this version:}

Jean-Christophe Géminard, C Laroche. Energy of a single bead bouncing on a vibrating plate: Experiments and numerical simulations. Physical Review E: Statistical, Nonlinear, and Soft Matter Physics, 2002, 10.1103/PhysRevE.68.031305 . hal-01238264

\section{HAL Id: hal-01238264 \\ https://hal.science/hal-01238264}

Submitted on 4 Dec 2015

HAL is a multi-disciplinary open access archive for the deposit and dissemination of scientific research documents, whether they are published or not. The documents may come from teaching and research institutions in France or abroad, or from public or private research centers.
L'archive ouverte pluridisciplinaire HAL, est destinée au dépôt et à la diffusion de documents scientifiques de niveau recherche, publiés ou non, émanant des établissements d'enseignement et de recherche français ou étrangers, des laboratoires publics ou privés. 


\title{
Energy of a single bead bouncing on a vibrating plate: Experiments and numerical simulations
}

\author{
J.-C. Géminard and C. Laroche \\ Laboratoire de Physique de l'ENS de Lyon, 46 Allée d'Italie, 69364 Lyon Cedex 07, France
}

(Received 14 December 2002; published 15 September 2003)

\begin{abstract}
The energy of a single bead bouncing on a vibrating plate is determined in simulations and experiments by tracking the bead-plate collision times. The plate oscillates sinusoidally along the vertical with the dimensionless peak acceleration $\Gamma$, and the bead-plate collisions are characterized by the velocity restitution coefficient $\epsilon$. Above the threshold dimensionless peak acceleration $\Gamma_{s} \simeq 0.85$, which does not depend on the restitution coefficient, the bead energy is shown to initially increase linearly with the vibration amplitude $A$, whereas it is found to scale like $v_{p}^{2} /(1-\epsilon)$, where $v_{p}$ is the peak velocity of the plate, only in the limit $\Gamma \gg \Gamma_{s}$. The threshold $\Gamma_{s}$ is shown to decrease when the bead is subjected, in simulations, to additional nondissipative collisions occurring with the typical frequency $\nu_{c}$. As a consequence, the bead energy scales like $v_{p}^{2} /(1-\epsilon)$ for all vibration strengths in the limit $\nu_{c} \gg \nu_{c}^{*}$. From the experimental and numerical findings, an analytical expression of the bead energy as a function of the experimental parameters is proposed.
\end{abstract}

DOI: 10.1103/PhysRevE.68.031305

PACS number(s): 81.05.Rm, 45.50.-j, 05.45.-a, 05.40.-a

As they exhibit a wide range of unusual behaviors, granular materials are the subject of intensive investigations [1-3]. Because of the inelastic nature of the contacts between the grains, these systems are intrinsically dissipative. In order to explore experimentally the effects of the dissipation on the properties of granular systems, it is convenient to produce stationary states; they are achieved by continuously providing the system with energy, compensating the energy intrinsically lost when the grains are in motion. Among these studies, one can note the experimental realization of twodimensional (2D) granular gases, consisting of inelastic beads constituting less than one-layer coverage on a vertically shaken, horizontal plate [4-8] (the experimental situation has been the framework of molecular-dynamics simulations by Nie et al. [9]). The velocity distributions, granular temperature, pressure, as well as phase transitions have been studied as functions of the vibration strength, usually characterized by the peak plate acceleration $\Gamma$. Nevertheless, detailed analysis of the clustering transition and of the pressure indicates that the vibrating boundary becomes inefficient to thermalize the system when the acceleration or the density of the gas are decreased. The energy input by the vibrating boundary has been the subject of several theoretical studies [10-13]; the scaling law for the energy as a function of the vibration strength has been shown to depend on the shape of the boundary vibration (sinusoidal, sawtooth, etc.) and on the nature of the dissipation within the gas (viscous, inelastic). In these studies, the assumption was made that the bead impinges randomly on the boundary; we note that this is not the case when the bead collides more than once with the boundary between two collisions with another bead. One can easily show that the velocity is exponentially correlated between two successive collisions in this case. It is hence relevant to study the dynamical behavior of a single bead bouncing on a vibrating plate, and to determine the mean energy $\langle E\rangle$ of the bead as a function of the vibration strength. The system has been widely studied, but, since the seminal work of Fermi [14], most of the studies focused on the period-doubling route to chaos [15-17]. Moreover, Warr et al., considering the situation both theoretically and experimentally, found a qualitative disagreement between their theoretical and experimental findings [18]; the mean energy of the bouncing bead was found to scale like the peak plate velocity $v_{p}$ in experiments whereas it was expected, theoretically and numerically, to scale like $v_{p}^{2}$. Experimentally, the velocity distributions and the mean energy were determined by tracking the bead with high speed photography. The theoretical scaling was found by writing a discrete-time Langevin equation. Because of the experimental method, only a small number of bounces could be analyzed. Inaccuracies due to air drag, small sample size, and human biases were thought to be largely responsible for this discrepancy. We present an alternative experimental method permitting the analysis of a large number of bounces, and hence making possible the determination of the velocity distributions and the energy with a better accuracy. A good agreement is found between the experimental results and a numerical simulation similar to that presented in Ref. [18]. In addition, the numerical study is extended in order to account for the effects of the collisions between beads within the gas.

Let us now consider the case of a single bead bouncing on a plate. The plate undergoes harmonic oscillations in the vertical direction according to $z(t)=A \cos (2 \pi \nu t+\phi)$, with $A$ the vibration amplitude and $\nu=\omega / 2 \pi$ the frequency. The energy dissipation, associated with the bead-plate collisions, is characterized by the velocity restitution coefficient $\epsilon$ (we assume, in the following, that $\epsilon$ does not depend on the impact velocity $[19,20]$, and neglect rolling or sliding frictions plausibly involved in the bead-plate contacts [21,22]).

We consider first the numerical simulation. For convenience, we normalize the lengths to the vibration amplitude $A$ and the times to the characteristic time $1 / 2 \pi \nu$. The bead, when not in contact with the plate, moves under the force of gravity according to

$$
\tilde{h}(t)=\widetilde{h}_{n}+\tilde{v}_{n}\left(\tilde{t}-\tilde{\tau}_{n}\right)-\frac{1}{2 \Gamma}\left(\tilde{t}-\tilde{\tau}_{n}\right)^{2},
$$

where $\widetilde{h}_{n}$ and $\widetilde{v}_{n}$ are, respectively, the initial dimensionless height and the velocity of the particle after the $n^{\text {th }}$ collision 
with the plate that occurred at $\tilde{\tau}_{n}$ (the dimensionless peak plate acceleration $\Gamma \equiv A \omega^{2} / g$, where $g$ denotes the acceleration due to gravity). The corresponding dimensionless energy of the bead (or dimensionless bouncing height) $H_{b}$ $=E / m g A$ is constant between two successive collisions with the plate and can be written as $H_{b}=\Gamma \tilde{v}^{2} / 2+\tilde{h}$. The velocity of the bead $\tilde{v}_{n}$ is given by

$$
\tilde{v}_{n}=(1+\epsilon) \tilde{v}_{p}-\epsilon \tilde{v},
$$

where $\tilde{v}$ is the bead velocity prior to the collision and $\tilde{v}_{p}$ $=-\cos \left(\tilde{\tau}_{n}+\phi\right)$, the plate velocity at $\tilde{\tau}_{n}$. The next collision time $\tilde{\tau}_{n+1}$ is the first root of $\tilde{z}\left(\tilde{\tau}_{n+1}\right)=\widetilde{h}\left(\tilde{\tau}_{n+1}\right)\left(\tilde{\tau}_{n+1}\right.$ $>\tilde{\tau}_{n}$ ). In order to handle chattering [16] or inelastic collapse [23], we stick the bead to the plate when the time interval between successive collisions $\delta \tilde{\tau}_{n} \equiv \tilde{\tau}_{n}-\tilde{\tau}_{n-1}$ is smaller than a cutoff time $\left(\delta \tilde{\tau}<10^{-4}\right)$. The bead then moves with the plate until the acceleration $\ddot{\widetilde{z}}(\tilde{t})$ is sufficient to release the particle into a new trajectory. We point out that the relation [Eq. (2)] between the dimensionless bead velocities before $(\tilde{v})$ and after $\left(\tilde{v}_{n}\right)$ collision with the vibrating plate depends only on the collision time $\tilde{\tau}_{n}$ and not on the characteristic amplitude $A$ and frequency $\nu$ of the plate vibration. Thus, it appears clearly that the only control parameter in the problem is the dimensionless acceleration $\Gamma$ that appears in Eq. (1).

In order to account for the effects of the bead-bead collisions (assumed to be nondissipative and to occur with the typical frequency $\nu_{c}$ ), when the vibrating plate is used as energy input for a granular gas, we allow the numerical procedure to renew the initial conditions of the bead, keeping the energy $E(t)$ unchanged. The main purpose of the model is to study the effects of the resulting loss of correlation between the particle motion and the plate vibration. We assume that the duration $\delta t$ of the free motion of the bead between two successive bead-bead collisions satisfies the probability distribution $\mathcal{P}(\delta t) \sim \exp \left(-\nu_{c} \delta t\right)$, where $\nu_{c}$ is the typical collision frequency. In the numerical simulation, one collision is accounted as follows: the phase $\phi$ of the plate motion is chosen randomly in the interval $[0,2 \pi[$ and the bead velocity $\tilde{v}$ randomly in the interval $\left[-\sqrt{2 H_{b} / \Gamma}\right.$, $\left.+\sqrt{2 H_{b} / \Gamma}\right]$. The corresponding bead height $\tilde{h}$ then satisfies $\tilde{h}=H_{b}-\Gamma \tilde{v}^{2} / 2$, so that the collision does not change the energy of the bead but only its time phase with respect to the plate.

The numerical results obtained for a bead bouncing freely $\left(\nu_{c}=0\right)$ are presented in Fig. 1. We find that the mean value over time, $\left\langle H_{b}\right\rangle$, of the dimensionless mean bouncing height depends linearly on $\Gamma$ according to $\left\langle H_{b}\right\rangle=(-\alpha+\beta \Gamma)$. Results obtained for different values of $\epsilon$ show that the slope $\beta \sim 1 /\left[(1-\epsilon)+b(1-\epsilon)^{2}\right]$ whereas, by contrast, the ratio $\Gamma_{s} \equiv \alpha / \beta$ does not to depend on the restitution coefficient $\epsilon$. As a consequence, the mean energy of the bead $\langle E\rangle$ $=m g A\left\langle H_{b}\right\rangle$ reads

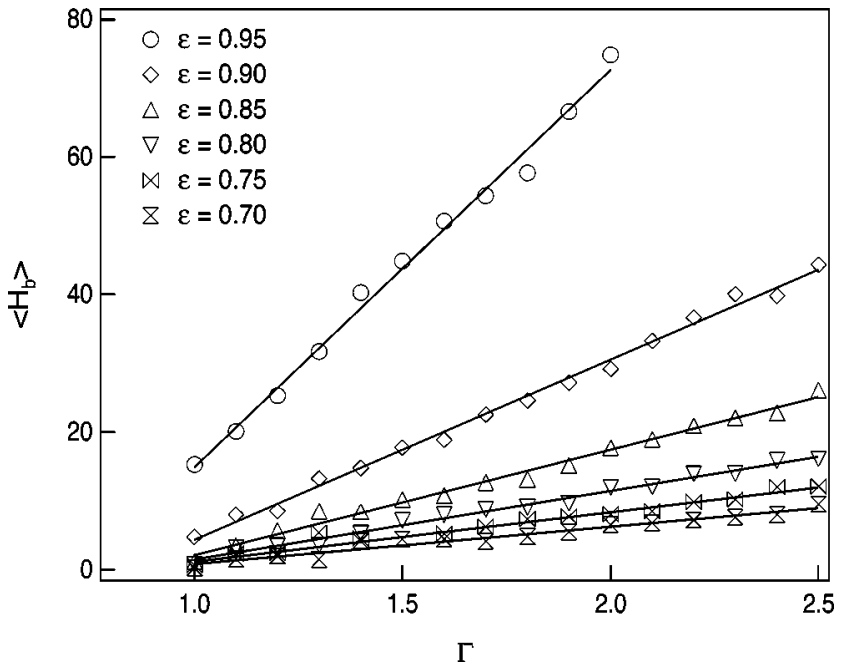

FIG. 1. Mean bouncing height $\left\langle H_{b}\right\rangle$ vs acceleration $\Gamma$. The trajectories are computed, for different values of the restitution coefficient $\epsilon$, during $10^{5}$ periods of the plate oscillation.

$$
\langle E\rangle=a \frac{m g^{2}}{\omega^{2}} \frac{\left(\Gamma-\Gamma_{s}\right) \Gamma}{(1-\epsilon)+b(1-\epsilon)^{2}},
$$

with the constant parameters $a=a^{0} \equiv 3.8 \pm 0.1, \quad b=4.45$ \pm 0.02 , and $\Gamma_{s}=\Gamma_{s}^{0} \equiv 0.85 \pm 0.01$. According to Eq. (3), the energy $\langle E\rangle$ scales like $v_{p}^{2}$ only when $\Gamma \gg \Gamma_{s}$, whereas it increases linearly with vibration amplitude $A$ when $\Gamma \simeq \Gamma_{s}$. Moreover, the results show that there exists a threshold dimensionless peak acceleration of the plate, $\Gamma_{s}$, above which the bead can experience a nontrivial trajectory; the mean energy of the bead, $\langle E\rangle$, is then given by Eq. (3).

We compared the numerical results, obtained in the case of the freely bouncing bead $\left(\nu_{c}=0\right)$, to experimental measurements. The experimental setup consists of one horizontal glass plate $\left(2 \times 2 \mathrm{~cm}^{2}\right)$ which is vibrated vertically. A glass plate is used in order to avoid any wear of the plate surface; indeed successive impacts of the bead at the same location on the surface of metallic plates always led to the formation of a visible small indent. The system is vibrated vertically with the help of an electromagnetic shaker (Brüel \& Kjaer, Type 4803) driven by sine waves $(\nu=40-80 \mathrm{~Hz})$ from a low distortion signal generator (Stanford Research Systems, DS345) and a power amplifier (Kepco, BOP50-4M). The amplitude $A$ of the vertical motion is measured with the help of an inductive sensor (Electrocorp, EMD1053) so that the peak plate acceleration $\Gamma \in[0,3]$ is known to within 0.01 . The bead (Marteau \& Lemarié, steel, diameter $D=10 \mathrm{~mm}$ ) is guided by a glass tube (inner diameter $10.1 \mathrm{~mm}$ ) so that it does not escape the system. The bottom edge of the glass tube is placed about $5 \mathrm{~mm}$ above the plate surface in order to avoid air to be trapped underneath the bead. An impact sensor (PCB-200B) is placed between the vibrator and the plate. The signal is recorded on a PC equipped with a data acquisition board (Data Translation, DT300), and the collision times $\tau_{n}$ are detected numerically. The energy of the bead is calculated from the successive collision times $\tau_{n}$ and from the corresponding vertical positions of the plate. Seeking for 


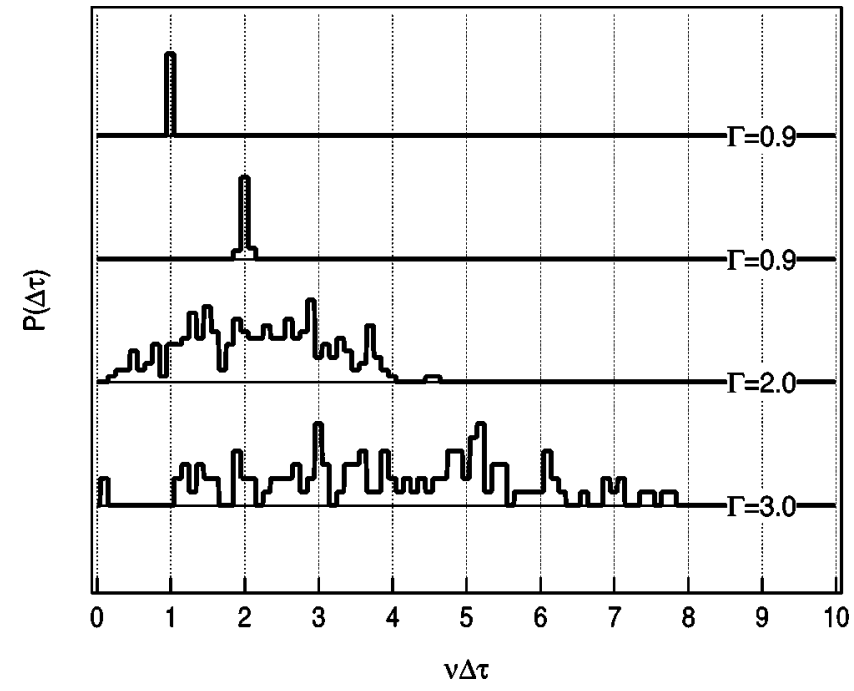

FIG. 2. Probability distribution $P(\Delta \tau)$ vs $\Delta \tau$. The synchronization of the bead to the plate motion $(k=1$ and $k=2)$ is pointed out by the two distributions at $\Gamma=0.9$. For the sake of clarity, the distributions are rescaled and shifted $(\nu=60 \mathrm{~Hz}$, sampling frequency is $60 \mathrm{kHz}$ during $8 \mathrm{~s}$ ).

simplicity, we neglect the vibration amplitude $A$ with respect to the bouncing height, and write the energy of the bead between $\tau_{n}$ and $\tau_{n+1}$ as

$$
E_{n}=\frac{m g^{2}}{8} \Delta \tau_{n}^{2},
$$

where $\Delta \tau_{n}=\tau_{n+1}-\tau_{n}$ stands for the flight duration with energy $E_{n}$. From the experimental distributions of $\Delta \tau_{n}$ (Fig. 2 ), the mean energy of the bead over time is estimated as

$$
\langle E\rangle=\frac{\sum E_{n} \Delta \tau_{n}}{\sum \Delta \tau_{n}}=\frac{m g^{2}}{8} \frac{\sum \Delta \tau_{n}^{3}}{\sum \Delta \tau_{n}} .
$$

We measure the mean energy of the bead $\langle E\rangle$ as a function of the dimensionless acceleration $\Gamma$ by changing the vibration amplitude $A$ at constant frequency $\nu$. The experimental measurements agree quantitatively with the numerical simulations at large acceleration (typically $\Gamma>1.4$ ) whereas departure from Eq. (1) is observed when the bead motion synchronizes to the plate oscillation (Fig. 3). In this case, the bead experiences periodic trajectories with $\Delta \tau_{n}$ $=k / \nu$ ( $k$ is an integer) associated with the constant energy

$$
E_{k}=m \frac{g^{2}}{\omega^{2}}\left(\frac{\pi^{2} k^{2}}{2}+\sqrt{\Gamma^{2}-\Gamma_{k}^{2}}\right),
$$

which depends only slightly on the plate acceleration $\Gamma$. Such a periodic trajectory can exist for accelerations larger than $\Gamma_{k}=k \pi(1-\epsilon) /(1+\epsilon)$. Due to synchronization of the bead motion to the plate oscillation, we observe hysteresis for small values of $\Gamma$ (Fig. 3). When the acceleration $\Gamma$ is decreased, the bead synchronizes to the plate motion for $\Gamma$ $\simeq 1.5$ and then experiences a periodic trajectory with $k=2$

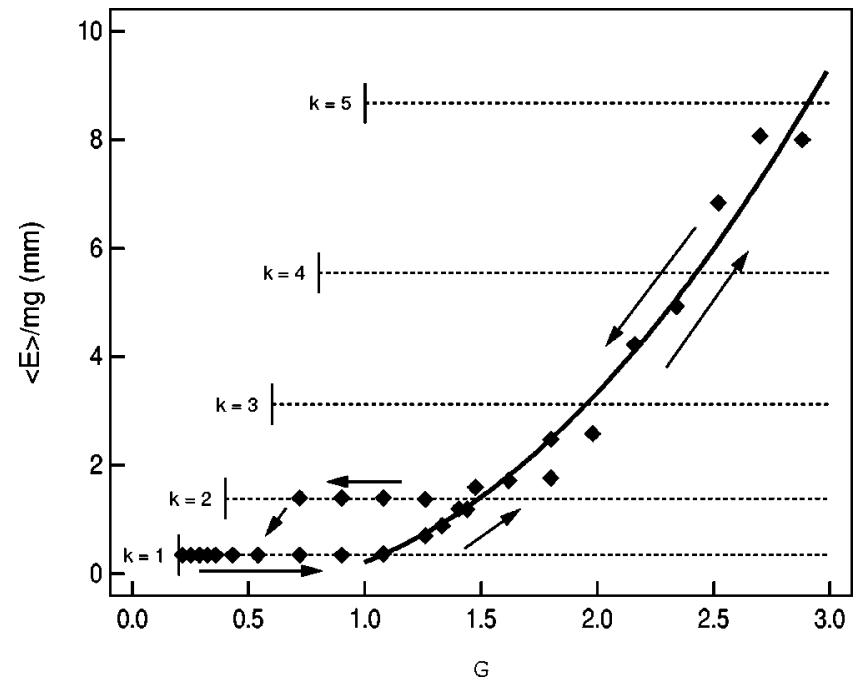

FIG. 3. Energy $\langle E\rangle$ vs acceleration $\Gamma$. Diamonds: experimental data ( $\nu=60 \mathrm{~Hz}$, sampling frequency is $60 \mathrm{kHz}$ during $8 \mathrm{~s}$ ). Dashed lines: energy of the periodic modes $E_{k}$. Vertical dashes: minimum acceleration $\Gamma_{k}$. Solid line: Eq. (3) with $\epsilon=0.88$.

down to $\Gamma \simeq 0.7>\Gamma_{2}$. For $\Gamma<0.7$, the bead experiences a periodic trajectory with $k=1$ down to $\Gamma_{1} \simeq 0.2$, below which the bead sticks to the plate. By contrast, when the acceleration $\Gamma$ is increased, the energy of the bead, which initially experiences the first periodic trajectory $(k=1)$, increases continuously according to Eq. (1) above $\Gamma>1.07$.

In order to evaluate the effect of the bead-bead collisions, we increased the frequency $\nu_{c}$ in the numerical simulations; the mean bouncing height $\left\langle H_{b}\right\rangle$ decreases at large acceleration whereas it increases at small accelerations (Fig. 4). This behavior results from a simultaneous decrease of the threshold acceleration $\Gamma_{s}$ and of the slope $a$. By contrast, the parameter $b$ remains unchanged within the scatter of the numerical data. The threshold acceleration $\Gamma_{s}$ decreases exponentially with increasing collision frequency $\nu_{c}$ according to

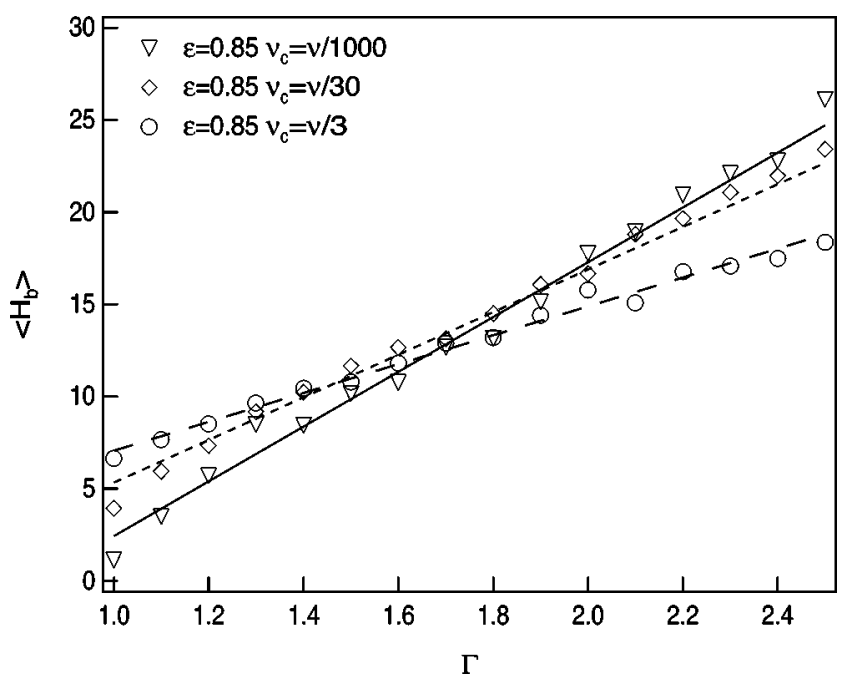

FIG. 4. Mean bouncing height $\left\langle H_{b}\right\rangle$ vs acceleration $\Gamma$. 


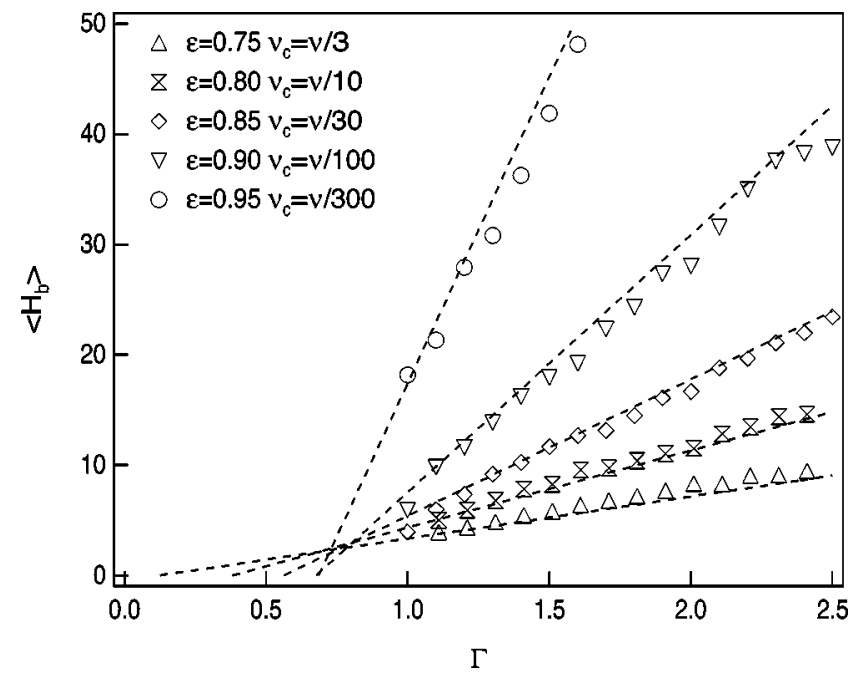

FIG. 5. Mean bouncing height $\left\langle H_{b}\right\rangle$ vs acceleration $\Gamma$. Symbols: numerical simulations. Dashed lines: Eq. (3) with $\Gamma_{s}$ [Eq. (7)] and $a$ [Eq. (8)].

$$
\Gamma_{s}=\Gamma_{s}^{0} \exp \left(-\frac{\nu_{c}}{\nu_{c}^{*}}\right)
$$

where the characteristic collision frequency $\nu_{c}^{*}=(1.4$ $\pm 0.3)(1-\epsilon)^{1.5 \pm 0.1} \nu$ depends on the restitution coefficient $\epsilon$ $\left[\Gamma_{s}^{0} \simeq 0.85\right.$ denotes the threshold dimensionless peak acceleration of the plate in the limit $\nu_{c} \rightarrow 0 \mathrm{Eq}$. (3)]. In the same way, the coefficient $a$ satisfies

$$
a=a^{*}+\left(a^{0}-a^{*}\right) \exp \left(-\frac{\nu_{c}}{\nu_{c}^{*}}\right)
$$

with $a^{*}=1.7 \pm 0.1$ and the same typical frequency $\nu_{c}^{*}$. The numerical data are successfully described to within $10 \%$ by Eq. (3) for $\epsilon \in[0.7,0.95]$ and $\Gamma \in[1.0,2.5]$ (Fig. 5). According to Eq. (3), in the limit $\nu_{c} \gg \nu_{c}^{*}$, the mean energy $\langle E\rangle$ $=a^{*} v_{p}^{2} /\left[(1-\epsilon)+b(1-\epsilon)^{2}\right]$ scales like $v_{p}^{2}$ for all accelerations $\Gamma$; when the typical frequency $\nu_{c}$ of the bead-bead collisions is large enough, the phase $\phi$ of the plate motion is not correlated between two successive bead-plate collisions. The random phase approximation is then fulfilled and the Langevin description is relevant. As a consequence, the energy $\langle E\rangle$ scales like $v_{p}^{2}$, as predicted theoretically by Warr et al. [18].

In conclusion, the numerical and experimental studies of the energy $\langle E\rangle$ of a single bead bouncing freely on a vibrating plate agree quantitatively. From the numerical data, we propose an analytical expression of the mean energy of the bead as a function of the experimental parameters. For all accelerations, the energy scales like $1 /(1-\epsilon)$ in the limit $\epsilon$ $\rightarrow 1$. Above the dimensionless threshold acceleration $\Gamma_{s}$ $\simeq 0.85$, which does not depend on the restitution coefficient $\epsilon$, the energy of the bead, experiencing a complicated motion, increases almost linearly with the vibration amplitude $A$. The energy scales like $v_{p}^{2}$, where $v_{p}$ is the peak plate velocity, only at large enough acceleration $\left(\Gamma \gg \Gamma_{s}\right)$. The threshold acceleration $\Gamma_{s}$ is shown to decrease when the bead is subjected, in simulations, to additional nondissipative collisions occurring with the typical frequency $\nu_{c}$. The bead energy scales like $v_{p}^{2} /(1-\epsilon)$ for all vibration strengths in the limit $\nu_{c} \gg \nu_{c}^{*}$, where $\nu_{c}^{*}$ is a characteristic collision frequency which depends on the bead-plate restitution coefficient $\epsilon$ and on the vibration frequency. These results are important clues for understanding the properties of vertically vibrated granular gases. For instance, the dependence of the mean energy of the bead on the bead-bead collision frequency $\nu_{c}$ could partly explain the hysteresis observed in the transition from the crystal to the gas phase in 2D granular gases. We plan to make use of the experimental method to determine accurately the probability distributions and correlation functions of the bead and plate velocities at the impact times. The results should help in writing a statistical theory depicting the dependence of the bead energy on the vibration strength, and then improving the understanding of the thermalization of granular materials by vibrating boundaries.

The authors wish to thank E. Levêque and T. Biben for helpful conversations.
[1] H.M. Jaeger, S.R. Nagel, and R.P. Behringer, Rev. Mod. Phys. 68, 1259 (1996).

[2] L.P. Kadanoff, Rev. Mod. Phys. 71, 435 (1999).

[3] H.J. Herrmann, S. Luding, and R. Cafiero, Physica A 295, 93 (2001).

[4] J.S. Olafsen and J.S. Urbach, Phys. Rev. Lett. 81, 4369 (1998).

[5] J.S. Olafsen and J.S. Urbach, Phys. Rev. E 60, R2468 (1999).

[6] W. Losert, D.G. Cooper, A. Kudrolli, and J.P. Gollub, Chaos 9 , 682 (1999).

[7] W. Losert, D.G. Cooper, and J.P. Gollub, Phys. Rev. E 59, 5855 (1999).

[8] J.-C. Géminard and C. Laroche (unpublished).

[9] X. Nie, E. Ben-Naim, and S.Y. Chen, Europhys. Lett. 51, 679 (2000)

[10] S. Warr, J.M. Huntley, and G.H. Jacques, Phys. Rev. E 52,
5583 (1995).

[11] J.M. Huntley, Phys. Rev. E 58, 5168 (1998).

[12] V. Kumaran, Phys. Rev. E 57, 5660 (1998).

[13] S. McNamara and S. Luding, Phys. Rev. E 58, 813 (1998).

[14] E. Fermi, Phys. Rev. 75, 1169 (1949).

[15] P. Pieranski, J. Phys. 44, 573 (1983).

[16] J.M. Luck and A. Mehta, Phys. Rev. E 48, 3988 (1993), and references therein.

[17] P. Devillard, J. Phys. I 4, 1003 (1994).

[18] S. Warr, W. Cooke, R.C. Ball, and J.M. Huntley, Physica A 231, 551 (1996).

[19] C.V. Raman, Phys. Rev. 12, 442 (1918); D. Tabor, Proc. R. Soc. London, Ser. A 192, 247 (1948); W. Goldsmith, Impact (Arnold, London, 1960); K. L. Johnson, Contact Mechanics (Cambridge University Press, Cambridge, 1985); L. Labous, 
A.D. Rosato, and R.N. Dave, Phys. Rev. E 56, 5717 (1997); G. Kuwabara and K. Kono, Jpn. J. Appl. Phys. Part 1 26, 1230 (1987).

[20] E. Falcon, C. Laroche, S. Fauve, and C. Coste, Eur. Phys. J. B 3, 45 (1998), and references therein.
[21] L. Kondic, Phys. Rev. E 60, 751 (1999).

[22] B. Painter and R.P. Behringer, Phys. Rev. E 62, 2380 (2000).

[23] S. Luding, H.J. Herrmann, and A. Blumen, Phys. Rev. E 50, 3100 (1994); S. Luding, E. Clement, A. Blumen, J. Rajchenbach, and J. Duran, ibid. 49, 1634 (1994). 